\title{
Modelling of snow avalanche dynamics: influence of model parameters
}

\author{
A.N. BOZHINSKIY \\ Laboratory of Snow Avalanches and Mudflows, Geographical Faculty, Lomonosov Moscow State University, \\ 119991 Moscow, Russia \\ E-mail: abozh@mail.ru
}

\begin{abstract}
The three-parameter hydraulic model of snow avalanche dynamics including the coefficients of dry and turbulent friction and the coefficient of new-snow-mass entrainment was investigated. The 'Domestic' avalanche site in Elbrus region, Caucasus, Russia, was chosen as the model avalanche range. According to the model, the fixed avalanche run-out can be achieved with various combinations of model parameters. At the fixed value of the coefficient of entrainment $m_{\mathrm{e}}$, we have a curve on a plane of the coefficients of dry and turbulent friction. It was found that the family of curves ( $m_{\mathrm{e}}$ is a parameter) are crossed at the single point. The value of the coefficient of turbulent friction at the cross-point remained practically constant for the maximum and average avalanche run-outs. The conclusions obtained are confirmed by the results of modelling for six arbitrarily chosen avalanche sites: three in the Khibiny mountains, Kola Peninsula, Russia, two in the Elbrus region and one idealized site with an exponential longitudinal profile. The dependences of run-out on the coefficient of dry friction are constructed for all the investigated avalanche sites. The results are important for the statistical simulation of avalanche dynamics since they suggest the possibility of using only one random model parameter, namely, the coefficient of dry friction, in the model. The histograms and distribution functions of the coefficient of dry friction are constructed and presented for avalanche sites Nos 22 and 43 (Khibiny mountains) and 'Domestic', with the available series of field data.
\end{abstract}

\section{INTRODUCTION}

The dynamic characteristics and parameters of deposit of an avalanche body can be determined by applying mathematical models. As a rule, two-parameter dynamic models including the coefficients of dry and turbulent friction are used (Bozhinskiy and Losev, 1987; Eglit and Revol, 1998). Recalculations for avalanche sites, where field data for avalanche events are available, allow the ranges of model parameters to be estimated (Buser and Frutiger, 1980; Martinelli and others, 1980). Hydraulic models were applied to five European avalanche sites to determine the sensitivity of the models to input parameters (Barbolini and others, 2000). It was shown that the dry friction coefficient is the predominant influence on avalanche run-out. However, the number of such avalanche sites is limited. Moreover, the calculated values of model parameters are often not regional, and their use for neighboring avalanche sites in the same region is not always justified. Another approach is statistical simulation of the avalanche process (Barbolini and Savi, 2001; Bozhinskiy and others, 2001; Ancey and others, 2004; Bozhinskiy, 2004; Meunier and Ancey, 2004). The model parameters are assumed random, with average values corresponding to recalculated estimations, based on field data. However, the laws and ranges of model parameter distributions are poorly known, so a more detailed investigation of the influence of model parameters on avalanche dynamics is needed. The earlier investigation was incomplete (Bozhinskiy, 2007): only general tendencies of change of output model characteristics (run-out, thickness of avalanche deposits, velocity and height of leading front) were illustrated by varying the model parameters. The purpose of this work is to research in depth the influence of the interaction of model parameters on output model characteristics, using a three-parameter dynamic model.

\section{MODEL}

The avalanche dynamics model used for research is characterized by three non-dimensional parameters: the coefficients $\mu$ and $k$ of dry and turbulent friction, respectively, and the coefficient $m_{\mathrm{e}}$ of new-snow-mass entrainment into the motion (Bozhinskiy and others, 2001). The system of model equations has the following form. The mass conservation equation is

$$
(H)_{t}+(H U)_{s}=q_{\mathrm{b}}
$$

and the momentum conservation equation is

$$
\begin{aligned}
(H U)_{t}+\left(H U^{2}\right)_{s}= & g H \sin \psi-\frac{g}{2}\left(H^{2} \cos \psi\right)_{s} \\
& -\mu g H \cos \psi-k U|U|,
\end{aligned}
$$

where $H$ and $U$ are the depth and velocity of the avalanche body, respectively, $\psi$ is the local slope angle, $q_{\mathrm{b}}$ is the rate of specific snow volume (per unit area of the bottom), $g$ is the gravitational acceleration, $s$ is the coordinate along the slope and $t$ is time. The parentheses with attached lower index designate partial derivatives with respect to the variable, indicated by this index. The avalanche density $\rho$ is assumed constant.

The specific volume of snow masses entrained into the motion is assumed to be proportional to the avalanche velocity

$$
q_{\mathrm{b}}=m_{\mathrm{e}} U \text {. }
$$

According to this phenomenological law of entrainment, the leading edge of the avalanche spreads over undisturbed snow cover, and additional snow mass is entrained by gradual shearing of the layer along the entire avalanche body. The other law characterizes the entrainment of snow peeled off by the avalanche at the leading edge. When the 


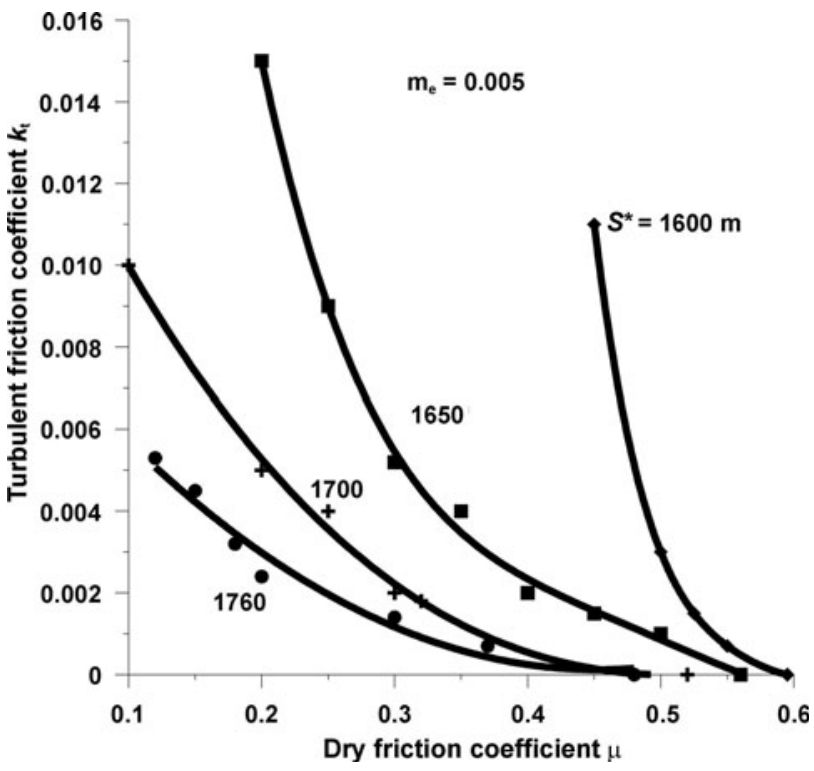

Fig. 1. Family of curves (run-out $S^{*}$ is a parameter) on the plane $k, \mu$; $m_{\mathrm{e}}=$ const.

law (Equation (3)) is used, the dynamic characteristics of an avalanche body near the leading edge change evenly (Bozhinskiy and Losev, 1987). Recently, different laws of snow entrainment have been analyzed, and it was shown that the dynamic characteristics of an avalanche body only weakly depend on the form of the entrainment law (Eglit and Demidov, 2005).

Finally, the snow-cover thickness $h$ during the avalanche motion on the slope diminishes according to

$$
h_{t}=-q_{\mathrm{b}}
$$

The deterministic model (Equations (1-4)) was used to obtain dynamic characteristics and parameters of avalanche deposits. It was also applied during statistical simulation of an avalanche process, when the initial data and the model parameters were considered as random (Bozhinskiy and others, 2001). The model acted as an operator. The output model characteristics were also found to be random, and the model captured the probabilistic contents.

\section{RESULTS AND DISCUSSION}

The 'Domestic' avalanche site in Elbrus region, Caucasus, Russia, was chosen as the model avalanche range. The snow-cover distribution along the slope was assumed uniform and equal to a mean observed value of $1.5 \mathrm{~m}$. Equations (1-4) were integrated numerically using the 'large-particle' numerical method, known in continuum mechanics (Belotserkovsky, 1984).

The fixed $\left(^{*}\right)$ avalanche run-out $S^{*}$, according to the model, can be achieved with various combinations of model parameters. When $m_{\mathrm{e}}=$ const., we have a curve on the plane $k, \mu$, which illustrates possible combinations of parameters of dry and turbulent friction ensuring achievement of the given run-out $S^{*}$ by model avalanche. For series values of $S^{*}$ we receive, by definition, the family of uncrossed curves (Fig. 1); the value of $m_{\mathrm{e}}=0.005$ corresponds to entrainment about a half of thickness of the snow cover on a slope. However, the situation changes when the third model parameter, $m_{\mathrm{e}}$, is taken into account. On the plane $k, \mu$, the

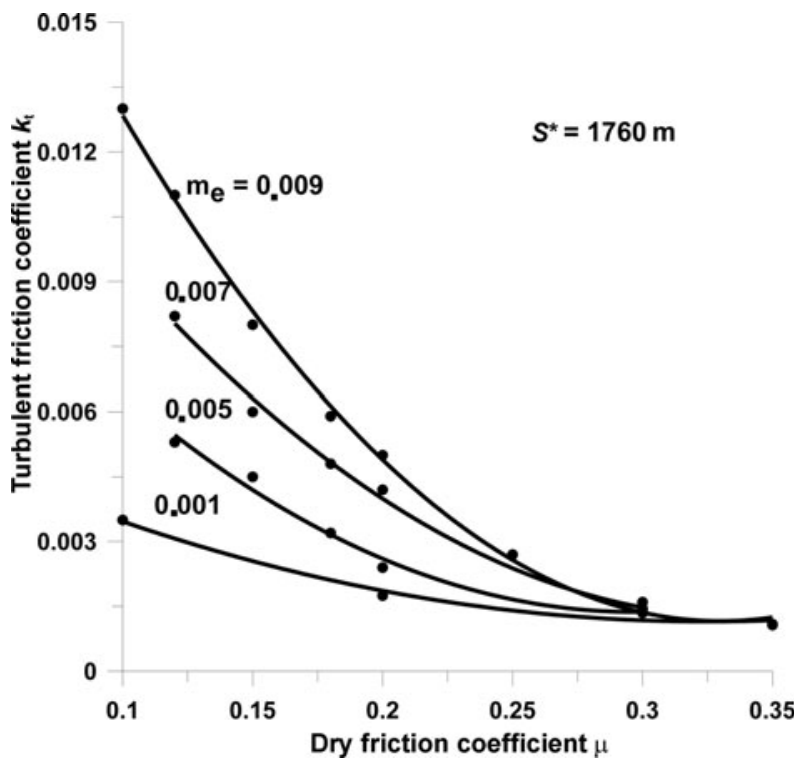

Fig. 2. Family of curves ( $m_{\mathrm{e}}$ is a parameter) on the plane $k, \mu$; $S^{*}=$ const.

family of curves ( $m_{\mathrm{e}}$ is a parameter) was constructed (Fig. 2). The curves were found to be crossed at the single point (Fig. 2). The minimum value, $m_{\mathrm{e}}=0.001$, corresponds to entrainment into an avalanche flow of no more than 0.1 of the initial thickness of a snow cover on a slope, and the maximum value, $m_{\mathrm{e}}=0.009$, corresponds to the complete entrainment of a snow cover into an avalanche flow. The existence of the cross-point for the $m_{\mathrm{e}}$-parameter family of curves means that there is a pair of values $k, \mu$ for which the avalanche run-out does not depend on new-snow-mass entrainment. It is known that the entrainment of new snow masses into an avalanche flow results in both increasing active force and additional resistance to motion (Bozhinskiy and Losev, 1987). The obtained value of $k$, corresponding to the cross-point, is small, resulting in suppression of turbulent resistance. At the same time, the active force and the dry friction force grow proportionally to increased mass of an avalanche body. Therefore, an independence of run-out on entrained masses takes place. Thus, when for determination of run-out the values of $k, \mu$ appropriating to the cross-point are used, the model appears as two-parametric, because the influence of snow-cover entrainment at such a combination of model parameters is practically excluded. However, if it is necessary to estimate thickness or volume of an avalanche body, the prescribing of a concrete value of the coefficient of entrainment $m_{\mathrm{e}}$ is essential.

A series of values of $S^{*}$ was further analyzed. Within the range of $400 \mathrm{~m}$ varying $S^{*}$ (maximum and average avalanche run-outs), the values of $k$, appropriate to cross-points, were practically identical. The effect of 'rapprochement' of curves, corresponding to minimum and maximum values of the coefficient of entrainment, with decreasing $k$ is also illustrated in Figure 3. It can be seen that when $k=0.0011$ the curves merge, and the single dependence of run-out on the coefficient of dry friction appears. The result obtained means that, for determination of run-out, it is possible to assume the value of the turbulent friction coefficient $k$ is constant and to vary only the coefficient of dry friction $\mu$. The curve illustrating the dependence of avalanche run-out on the coefficient of dry friction is shown in Figure 4. The 


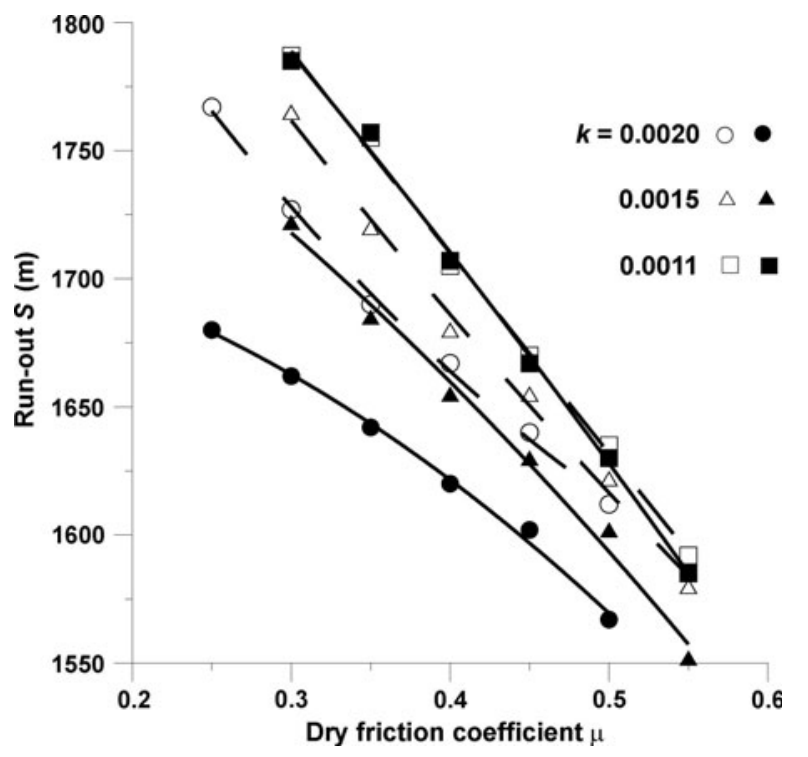

Fig. 3. Run-out vs dry friction coefficient for the 'Domestic' site; $k$ is a parameter. Closed symbols, solid lines: minimum $m_{\mathrm{e}}$; open symbols, dashed lines: maximum $m_{\mathrm{e}}$.

approximation of this dependence appears to be close to linear. Similar dependence of the observed run-outs on $\mu$ was obtained by Naaim and others (2004). A weak influence of $k$ within the range $0.01<k<0.025$ on a family of $S(\mu)$ was found using the rigid-body model of avalanche dynamics with two friction coefficients (Ancey and others, 2004).

The result obtained is important for the statistical simulation of avalanche dynamics, because it is possible to assume only one random model parameter $\mu$. The constant parameter of turbulent friction $k \approx 0.004$ and random parameter $\mu$ during statistical simulation were used in the two-parameter dynamic model (Barbolini and Savi, 2001).

Similar analysis of the influence of dynamic model parameters on the output characteristics of avalanches was carried out for other, arbitrarily chosen, avalanche sites:

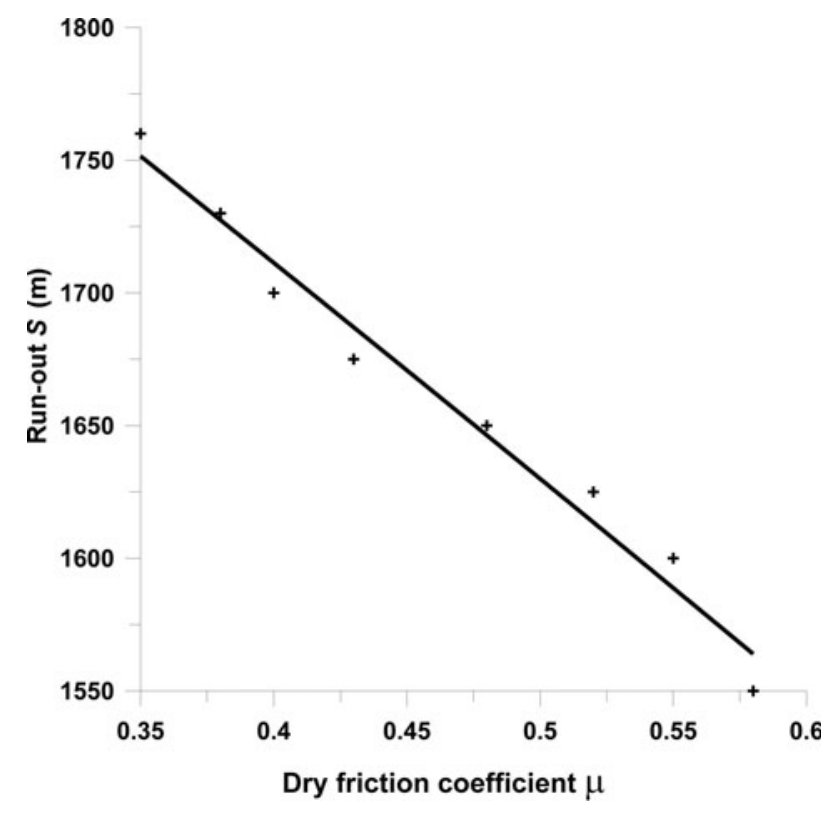

Fig. 4. Run-out vs dry friction coefficient for the 'Domestic' site; $k$ corresponds to cross-point.

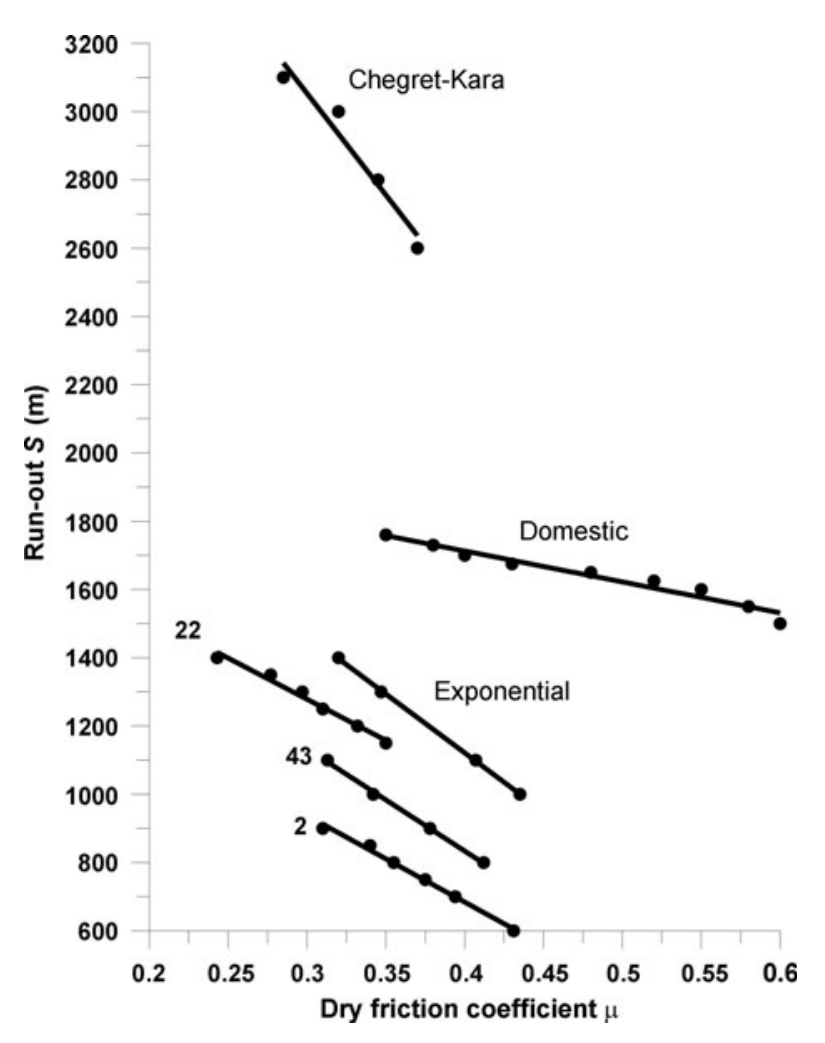

Fig. 5. Run-out vs dry friction coefficient for six avalanche sites.

Nos 2, 22 and 43 (Khibiny mountains, Kola Peninsula, Russia), Cheget-Kara (Elbrus region) and an idealized avalanche site with an exponential longitudinal profile. The results are shown in Table 1 , where the value of the coefficient of turbulent friction $k$ corresponding to the crosspoint of curves is given.

The values of parameter $k$ at the cross-point are very small for all the avalanche sites, testifying to the relatively weak turbulent mixing of snow into the modeled avalanche flow. However, this does not mean that the turbulent resistance can be neglected, because, for example, for the 'Domestic' avalanche site, the difference of run-outs when $k=0$ and $k=0.0011$ is within the $100-200 \mathrm{~m}$ range. For every site, the dependences of run-out on the coefficient of dry friction are constructed (Fig. 5). These dependences were approximated by linear functions $S=A+B \mu$. The values (in meters) of the coefficients of equations are given in Table $2 ; R$ is the coefficient of correlation.

The obtained dependences are valid within the range of change of $S$, including the maximum and average run-outs. For small values of $S$, the model coefficient of turbulent

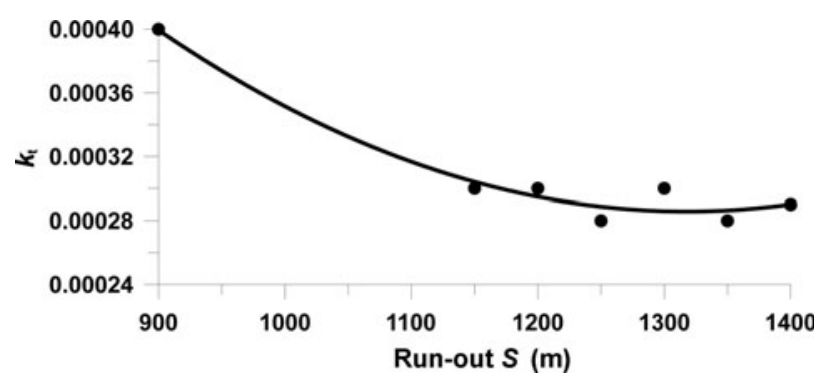

Fig. 6. Turbulent friction coefficient corresponding to cross-point vs run-out for avalanche site No. 22, Khibiny mountains. 


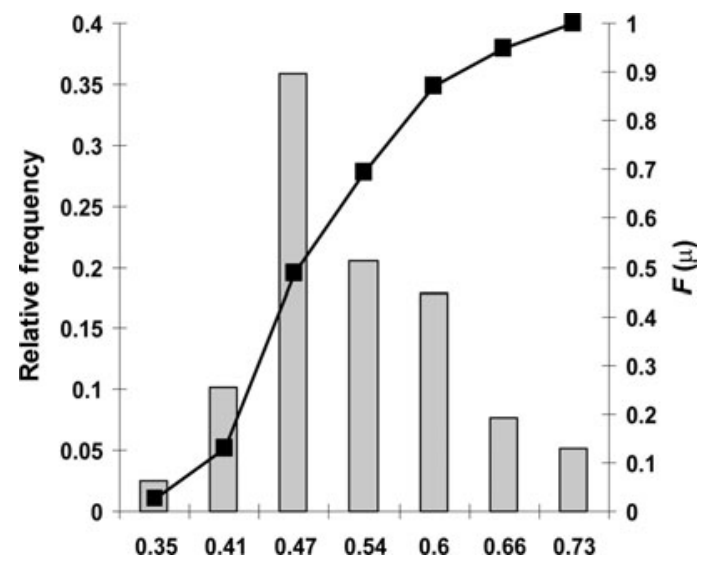

Fig. 7. Histogram and distribution function of dry friction coefficient for 'Domestic' avalanche site.

friction, appropriate to the cross-point, increases slightly. This is related to the fact that such avalanches stop on relatively steep segments of a slope, and it is practically impossible to create resistance to movement of an avalanche on these segments due to dry friction only. Therefore, turbulent friction, which is weak on flatter segments, should grow. Then the drag and stop of an avalanche body is possible. The example of dependence of $k$ at the cross-point for ranges of change of $S$, including relatively short run-outs, is given in Figure 6 . The significant range of $S$ within which $k \approx$ const. is noticeable.

Available field data of run-outs for the avalanche sites 'Domestic' (39 events), No. 22 (30 events) and No. 43 (24 events) and the received linear dependences $S(\mu)$ allow distributions of the model parameter $\mu$ to be constructed. The histogram and distribution function of $\mu$ are given for the 'Domestic' avalanche site in Figure 7. Characteristic statistics of distributions are given in Table 3.

The distribution of $\mu$ for the 'Domestic' and No. 22 avalanche sites is close to normal, though a positive kurtosis and skewness are available. Note that as the dependences of $\mu(S)$ are linear, then $M_{\mu}=a+b M_{\mathrm{s}}$ and $\sigma_{\mu}=|b| \sigma_{s}$, where $a$ and $b$ are the coefficients of linear dependence. As the coefficients of skewness and kurtosis are proportional to the third and fourth central moments, respectively, it is obvious that $\lambda_{\mathrm{a} \mu}=-\lambda_{\mathrm{as}}$ and $\lambda_{\mathrm{e} \mu}=\lambda_{\mathrm{es}}$. It follows that the coefficient $b$ is negative and the third moment is proportional to the cube, and the fourth moment to the fourth degree, of $b$ (Ventsel, 1969). Therefore, the mode and the tail of distribution of run-out shift to the right and left, respectively; the distribution of dry friction coefficient is the reverse (Fig. 7). For avalanche site No. 43, the distribution is close to uniform, there is almost no skewness, but a large negative kurtosis testifies the flatness of distribution.

\section{CONCLUSIONS}

The obtained dependences $S(\mu)$ should be considered as the property of an avalanche site. Application of the threeparameter avalanche dynamic model allows the value of the turbulent friction coefficient to be estimated and the dependence of run-out on the coefficient of dry friction to be constructed. Knowledge of the longitudinal profile and the distribution of a snow-cover thickness at the avalanche site is only needed to determine the value of the turbulent
Table 1. Values of the coefficient of turbulent friction corresponding to the cross-point

\begin{tabular}{|c|c|c|c|c|c|c|}
\hline \multirow{2}{*}{$\begin{array}{l}\text { Avalanche } \\
\text { site }\end{array}$} & \multicolumn{2}{|c|}{ Elbrus region } & \multicolumn{3}{|c|}{ Khibiny mountains } & \multirow{2}{*}{$\begin{array}{l}\text { Exponential } \\
\text { slope } \\
\text { (idealized) }\end{array}$} \\
\hline & Domestic & Cheget-Kara & 2 & 22 & 43 & \\
\hline$k$ & 0.0011 & 0.0005 & 0.000 & 0.000 & 0.0003 & 0.0010 \\
\hline
\end{tabular}

Table 2. Values of the coefficients of equations

\begin{tabular}{lccc}
\hline Avalanche site & $A$ & $B$ & $R^{2}$ \\
\hline 'Domestic' & 2074 & -905 & 0.96 \\
No. 2 & 1699 & -2536 & 0.99 \\
No. 22 & 2001 & -2410 & 0.98 \\
No. 43 & 2033 & -2997 & 0.99 \\
Cheget-Kara & 4838 & -5949 & 0.95 \\
Exponential slope & 2500 & -3447 & 0.99 \\
\end{tabular}

Table 3. Statistics of distributions of $\mu . M$ is the mathematical expectation, $\sigma$ is the root-mean-square deviation, $\lambda_{\mathrm{a}}, \lambda_{\mathrm{e}}$ and $C_{\mathrm{v}}$ are the coefficients of skewness, kurtosis and variation, respectively, and max and min are the maximum and minimum values, respectively

\begin{tabular}{llcc}
\hline Statistic & \multicolumn{3}{c}{ Avalanche site } \\
& Domestic & No. 22 & No. 43 \\
\hline$M$ & 0.5 & 0.36 & 0.41 \\
$\sigma$ & 0.09 & 0.06 & 0.07 \\
$\lambda_{\mathrm{a}}$ & 0.6 & 0.7 & -0.11 \\
$\lambda_{\mathrm{e}}$ & 0.53 & 0.73 & -1.0 \\
$\max$ & 0.73 & 0.53 & 0.52 \\
$\min$ & 0.35 & 0.26 & 0.29 \\
$C_{\mathrm{v}}$ & 0.18 & 0.17 & 0.16 \\
\hline
\end{tabular}

friction coefficient. This means that the model can be used for poorly investigated avalanche sites. Further development can provide a simplified statistical simulation of snow avalanche dynamics, since it is possible to use only one random model parameter (the coefficient of dry friction) to determine an avalanche run-out. It may be possible to construct distributions of the dry friction coefficient on the basis of available field data and statistical simulation for well-investigated avalanche sites.

\section{REFERENCES}

Ancey, C., C. Gervasoni and M. Meunier. 2004. Computing extreme avalanches. Cold Reg. Sci. Technol., 39(2-3), 161-180.

Barbolini, M. and F. Savi. 2001. Estimate of uncertainties in avalanche hazard mapping. Ann. Glaciol., 32, 299-305.

Barbolini, M., U. Gruber, C.J. Keylock, M. Naaim and F. Savi. 2000. Application of statistical and hydraulic-continuum dense-snow avalanche models to five real European sites. Cold Reg. Sci. Technol., 31(2), 133-149. 
Belotserkovsky, O.M. 1984. Chislennoe modelirovanie v mekhanike cploshnoy cpdey [Numerical modelling in continuum mechanics]. Moscow, Science. [In Russian.]

Bozhinskiy, A.N. 2004. The Monte Carlo simulation of avalanchetype processes. Ann. Glaciol., 38, 351-356.

Bozhinskiy, A.N. 2007. On statistical simulation of snow avalanches dynamics. In Proceedings of the Third International Conference on Avalanches and Related Problems, 4-8 September 2006, Kirovsk, Russia. Apatity, Apatit Media $11-17$.

Bozhinskiy, A.N. and K.S. Losev. 1987. Osnovy lavinovedeniya [Fundamentals of avalanche science]. Leningrad, Gidrometeoizdat. [In Russian.]

Bozhinskiy, A.N., A.N. Nazarov and P.A. Chernouss. 2001. Avalanches: a probabilistic approach to modelling. Ann. Glaciol., 32, 255-258.

Buser, O. and H. Frutiger. 1980. Observed maximum run-out distance of snow avalanches and the determination of the friction coefficients $\mu$ and $\xi$. J. Glaciol., 26(94), 121-130.
Eglit, M.E. 1998. Mathematical modelling of dense avalanches. In Hestnes, E., ed. 25 Years of Snow Avalanche Research at NGI. Proceedings of the Anniversary Conference, 12-16 May 1998, Voss, Norway. Oslo, Norwegian Geotechnical Institute, 15-18. (NGI Publication 203.)

Eglit, M.E. and K.S. Demidov. 2005. Mathematical modeling of snow entrainment in avalanche motion. Cold Reg. Sci. Technol., 43(1-2), 10-23.

Martinelli, M., Jr, T.E. Lang and A.I. Mears. 1980. Calculations of avalanche friction coefficients from field data. J. Glaciol., 26(94), 109-119.

Meunier, M. and C. Ancey. 2004. Towards a conceptual approach to predetermining long-return-period avalanche run-out distances. J. Glaciol., 50(169), 268-278.

Naaim, M., F. Naaim-Bouvet, T. Faug and A. Bouchet. 2004. Dense snow avalanche modeling: flow, erosion, deposition and obstacle effects. Cold Reg. Sci. Technol., 39(2-3), 193-204.

Ventsel, E.S. 1969. Teoriya veroyatnostey [Probability theory]. Moscow, Gostechizdat. [In Russian.] 